\title{
The Conventional Braille Display State of the Art and Future Perspectives
}

\author{
Dipl.-Ing. Bernhard Stöger and Mag. Klaus Miesenberger \\ Educational endeavour "Computer Science for the Blind" \\ Johannes Kepler Universität \\ Altenbergerstr. 69, A-4040 Linz
}

\begin{abstract}
The conventional one-line Braille display is explained and discussed in great detail, the emphasis being laid on the ergonomic and software-technical aspects rather than the electronic or the electromechanical ones. Especially closely studied are the deeper aspects of text-based Braille display operation such as video attribute viewing and lightbar handling. In addition, some suggestions to improve present Braille display software are given, especially concerning the recently developed programs to access graphical user interfaces.
\end{abstract}

\section{What is Braille?}

The Frenchman Louis Braille (1809-1852) invented a writing system that is used by blind and visually impaired people and that is based on tactile rather than on optical perception. In original Braille, characters are generated by embossing small raised dots into a sheet of paper. Every Braille character occupies a small section of the sheet that is organized as a 2.3 grid and that can therefore take up to 6 dots. Every character (letter, number, punctuation mark etc.) is a certain selection within such a 2.3 arrangement. If one includes the space - the character with no dot at all -, one thus obtains 2 to the 6 th $=64$ possible characters; it is therefore possible to represent the letters of the Roman alphabet together with the numbers, the punctuation marks and several special characters.

Since its invention toward the end of the first half of the past century, Braille has been used by blind and strongly visually impaired people in two main ways: Firstly, special machines were devised that allowed a blind person to make personal notes this made possible written communication between blind persons, however, at least in general, without the possibility to include sighted people. Secondly, by special printing methods literature was produced in Braille.

\section{What is a Braille Display?}

When, by the end of the seventies and in the beginning of the eighties, the computer became more and more the central medium of information processing in all areas of society, attempts were made to make the computer accessible for the blind by means of the Braille system. In these efforts, the conventional way to write Braille dots on paper proved totally inadequate; for most of the time the computer is operated interactively, presenting much information to the user that is relevant only for the 
moment. The problem was to devise an output medium for the blind user that replaced the screen, the "refreshable display" used by the sighted. To this end, in the middle seventies electromagnetic display elements were developed. Each display element was able to move a small metal pin up and down between two positions, whose distance was about 1 millimeter. Every pin is furnished with a soft plastic top that can be felt as a Braille dot when in the raised position. Six such elements are arranged in a 2.3 grid, thus forming the matrix for a complete Braille character. Such a unit consisting of 6 or 8 (see below) pins together with the electromechanical elements for their motion is also referred to as a Braille module.

Theoretically, it would be possible to form a "tactile screen" from such Braille modules by arranging them in a rectangular manner, e.g., in a 25.80 matrix. However, both the electromagnetic and the later developed piezo electric modules are so expensive that a machine consisting of more than 80 modules is practically unaffordable. Until the time when a cheaper technology will be available, one must therefore be satisfied with representing one, one half, or even only one quarter of a line at a time. Such a machine consisting of 20,40 , or 80 linearly arranged Braille modules is called a Braille Display.

As mentioned above, the elements of a Braille display are arranged in a horizontal row. In most cases, to the left of this row there is a smaller row consisting of 3 or 4 Braille modules called Status Modules. These extra modules represent some additional information about the status of the display in encoded form, such as the present position of the Braille Window on the screen (see next section) or the position of the cursor.

Most Braille displays have a flat design. If they consist of 40 modules, their width is that of a PC keyboard. The depth is normally such that one can position the keyboard onto the display, the surface with the tactile pins being in front of the keyboard. From this ergonomically very advantageous connection between display and keyboard many sighted people gain the impression that the blind user would work with a special keyboard for the blind. However, this is completely wrong: Offthe-shelf PC keyboards are normally well suited for use by a blind person, the special thing is only the display.

There is a strong trend to design Braille displays in a portable form (weight less than 2 Kilogram). Since the dimensions of a notebook PC are much the same as the dimensions of a PC keyboard, one can position such a computer on top of the display. A combination of this kind, although already somewhat bulky and heavy, can still be considered portable, thus making the computer fully available to a blind user even when (s)he is on tour. The rechargeable batteries built into a portable Braille display grant 3 to 4 hours of independent operation, a time generally longer than that granted by the batteries of a notebook PC.

\section{The Braille Window. 40 vs. 80 Character Braille Display}

The blind computer user working with a Braille display never sees the whole screen at one glance: (s)he is limited to one line, in many cases only one half or a quarter of a line of the screen at a time. For sighted people it is hard to imagine that under such 
restricted conditions efficient use of modern software is still possible, and, yet, it is: The art is to move the Braille Window, that is, the one-dimensional portion of the screen presently displayed by the Braille modules, quickly and efficiently across the screen to places where relevant information is located. Such movements of the Braille display can be performed in two different ways:

User-controlled movement: On every Braille display the user finds certain keys (or key combinations) to move the Braille window line by line or, on a 40 character Braille display, half line by half line.

Software-controlled movement: Although the above mentioned functions allow complete access to every spot on the screen, work would be extremely cumbersome if the user were not supported by a set of automatic jump functions. The by far most important capability of this kind is the Cursor Binding function discussed in the next section.

\section{How the Cursor is Handled by a Braille Display}

There is probably no interactive, text-oriented software program that would not in the one or in the other form make use of the Cursor. Although today there are more and more situations where the cursor is replaced by a lightbar (see section 8.), the cursor still plays an essential part on the screen: It is present wherever text has to be input by the user. To deal with the cursor correctly is therefore an absolute necessity for every Braille display.

As soon as the Braille Window covers a portion of the screen containing the cursor, the actual position of the cursor is displayed by the corresponding Braille module. To do this, there are several different ways in use:

Display by the Full Braille Cell: The module corresponding to the cursor position shows the Braille character consisting of all eight dots.

Representation by Dot 7 and Dot 8: These two dots are added to the character at the cursor position.

Representation by Vibrating Cells: Some or all dots forming the character at the cursor position are put into a vibrating state.

Associated with the cursor are two important functions, one of which usercontrolled, the other software-controlled:

Jump to the Cursor: This user-controlled function lets one move the Braille window instantly to the cursor position, whatever its original position may have been. This becomes important if one wants to convince oneself about the present cursor position, or if the surroundings of the cursor on screen are to be explored.

Automatic Cursor Binding: This software-controlled capability moves the Braille window automatically to the cursor whenever its position on the screen changes. One consequence of this function, at first sight a rather trivial one, is the fact that, whenever the user inputs text from the keyboard, then the Braille Window is at the position of the input text, loosely speaking, it follows the input.

There are circumstances where automatic Cursor Binding has disturbing effects: These are situations where the screen is updated continuously and where a piece of 
information apart from the cursor has to be watched, whereas the cursor itself moves constantly on the screen.

\section{Cursor Routing}

In order to correct text written by any editor it is necessary to quickly move the cursor to a place where an error is found. Since the error was detected using the Braille window, the task is to move the cursor to a certain spot covered by the Braille window. It would be rather cumbersome if one had to use the cursor arrow keys to perform such a movement. In order to ease the work, most Braille displays are furnished with special buttons to perform what is called "Cursor Routing": Above every Braille module there is a knob which, when pressed, causes the cursor to move to that point on the screen presently covered by the Braille module. This mechanism which, in a sense, can be viewed as a tactile substitute for the mouse, makes it possible to easily correct texts processed by a computer.

Cursor routing keys exist both as mechanical buttons and as optical sensors. Sensors have the advantage that they require absolutely no physical pressure, but their disadvantage is that one can trigger unwanted actions by inadvertently touching them. This drawback does not appear with the mechanical variant, but there are some cases where very firm pressure is needed to trigger the cursor routing buttons. Another crucial advantage of the sensor variant is pointed out in the next section.

That the cursor can be routed only to those spots on the screen where it is allowed by the current application program is obvious. However, with some less developed implementations there are situations where a cursor routing cannot be performed although it would be allowed by the running application.

\section{Representation of video attributes}

The text characters and the position of the cursor are not the only items of information that are relevant within a text-oriented environment: An essential component are the video attributes that are assigned to every character on the screen. From the software-technical point of view, a video attribute is just a sequence of 8 bits that is associated with every screen character in addition to the 8 text bits. From the user's viewpoint, these attributes are visible in terms of foreground and background colors and of certain highlightings such as blinking, underlined or boldface characters. Hereby, the special form of an attribute is often of little relevance for a blind user. Rather, it is important to notice changes in attribute within a text and to grasp the information for whose sake the change was performed.

For the representation of attributes by means of a Braille display, there are several common possibilities:

Binary Representation: The Braille display is switched into a special attribute mode where every Braille module represents the attribute bits rather than the text bits of the corresponding character. Here, in contrast to the customs in the Braille writing system, one normally establishes a one-to-one correspondence between the bits of an attribute and the dots of the representing Braille cell. Since each of these bits represents a unique foreground colour, background colour or highlighting, the 
blind user can feel the actual colours or highlightings from the bit pattern displayed on the Braille line.

Encoded representation by the status modules (see section 2): With displays that have cursor routing buttons sometimes a special mode can be enabled where the attribute of a character is displayed by the status cells once the cursor routing button above this character is pressed or touched, respectively. For this representation, a code is employed that uses easily remembered, three or four letter abbreviations for the colours and the highlightings.

Representation of Certain Attributes by Using Additional Braille Dots: In most applications, only very few attributes are relevant to the user: In this case (and in similar cases) some Braille displays allow to define the relevant attribute as a "special attribute" and to have such a special attribute represented by adding dots 7 and 8(or dot 8 alone) to each character with the attribute in question.

\section{Handling Lightbars (Soft(ware) Cursors)}

The system cursor (or hardware cursor) discussed in section 4 is not the only object on the screen that has to be automatically followed by the sighted user's eyes (and hence also by the Braille display).In modern application programs, selections in pulldown menus, in listings like directories etc. are very often marked by a video attribute (a lightbar) rather than by the system cursor. If one of the arrow keys is pressed, then the position of the video attribute in question changes on screen, and the selected item can be viewed.

Theoretically, a Braille display user can use the methods discussed in the previous section to detect the presence of a lightbar attribute. However, efficiency and speed in working with modern software would dramatically decrease if such a method were applied. It would be desirable that, whenever an arrow key is pressed, then the Braille window automatically jumps to the place where the lightbar attribute is located, establishing an automatic following mechanism completely analogous to the cursor binding discussed in section 4 .

Contrary to the system cursor which is also referred to as the Hardware Cursor, spots of the screen carrying a lightbar are called Soft Cursors (or Software Cursors) in the world of blind users. The addressed problem will therefore be referred to as the Soft Cursor Problem.

Before we attempt to sketch the presently available methods to cope with the problem, we must remark that there is hardly a system on the present market that would solve the problem in a completely satisfactory way. Although today's Braille displays all are able to follow every soft cursor, it either requires much configuration or even programming work to install the mechanism, or it can be easily installed, but it causes trouble to switch to an alternative method of cursor following within the current application. It is certainly not exaggerated when we call the soft cursor problem the canonical weakness of the present Braille display.

The reason why different methods of soft cursor following were developed is because the spot on the screen where a lightbar attribute appears is not always unique, such that an algorithm to decide which occurrence should be displayed is 
needed. The most important example is the classical pull-down menu as is commonly used within the Microsoft and Borland programs. If, for example, in Microsoft Word no submenu of the horizontal menu "file - process -layout -insert ..." is selected, then the place where the highlight attribute is located is still unique it only moves across the top line of the screen when the left or right arrow key is pressed. However, as soon as one of the menu items, say "File", is selected, then the attribute appears not only on the "File" selection, but also on one of the vertically arranged sub-menus "new- open - close - ..." and so on.

The following strategies to overcome this problem were developed:

\subsection{Movement-oriented Soft Cursor Handling}

With this method, the user first defines the attribute to be followed. When the application in question is running, the (normally unique) place on the screen where the attribute has moved is calculated in regular time intervals, and the Braille window is directed to that location. For instance, in our above example of the pulldown menu, this method always presents that occurrence of the lightbar attribute currently relevant for the user: For in the first case where still no submenu is open, hitting a horizontal arrow key can cause a movement of the soft cursor only within the horizontal menu line, whereas in the second case where a vertical submenu is selected, hitting a vertical arrow key leaves the horizontal line unchanged, and a movement of the soft cursor attribute takes place only within the vertical section where the submenu resides.

The movement-oriented method is very simple to install, and in most cases it provides the desired results immediately. However, in order to use it efficiently, it has to switch back automatically to the normal cursor binding (section 4) in cases where a lightbar to follow is no longer present on the screen. Moreover, it should be possible to switch automatically between different attributes for movement-oriented lightbar handling. However, both automatisms are presently implemented within very few systems. Since in many applications situations where a soft cursor has to be followed and situations where the hardware cursor is relevant coexist and since very often more than just one lightbar attribute has to be watched, the afore-mentioned drawback kills much of this method's attractivity and efficiency.

\subsection{Window-oriented Monitoring}

Here the problem of multiple occurrence of the same attribute is faced by confining the monitoring process to a rectangular portion of the screen, a so-called "Window", within which the attribute occurs only once. The main disadvantage of this approach is apparent: The above stated need for automatic switching mechanisms appears here already in situations where the first method still works perfectly without them: In order to stick to our example: In the first case (no sub-menu open), the soft cursor window can be the entire screen. In the second case, however, when a sub-menu is opened, we shall exclude the top line of the screen in order to attain uniqueness. So, already in this simple situation, we need two different soft cursor windows, and we 
need an automated mechanism to switch between them unless we want to kill ease of working by an endless series of key hits for manual switching.

Especially within systems that utilize the third method, automatic switching procedures are already quite common. The idea behind the mechanisms is that the system regularly monitors the apparition of specified strings on the screen which are unique indicators for the necessity of an automatic switching. However, implementations of this kind have three crucial disadvantages:

- Complicated Installation

- Noticeable Loss of Performance

- High Memory Consumption

The problems pointed out above can probably be completely solved only by furnishing the Braille display software by artificial intelligence that analyses the Video Memory in order to automatically manage lightbar handling. Although there are already a few systems with such a software, it does not yet belong to the set of standard equipment for a Braille display.

\section{Computer Systems Accessible with a Braille Display}

Since the (IBM)-compatible PC is by far the most common computer in modern working life, Braille displays have been devised almost exclusively for this computer platform. Since, however, the PC can be used as a terminal for a vast variety of computer systems by terminal emulation software, the Braille display opens, via the PC as a connecting link, access to virtually every text-oriented computing environment to a blind user. Workstations with a UNIX (or a similar) operating system, VAX Computers, or mainframes with VM or MVS as their operating systems are only a few examples.

\section{Attaching a Braille Display to a Computer; Hardware and Software Solutions}

To attach a Braille display to a computer, there are two completely different ways:

\subsection{The Software Solution}

This is the more common variant: Here a TSR program is loaded in the PC that manages the monitoring of the Video Memory and the control of the Braille display, and the display is connected to the computer via the parallel or a serial port. The advantages of the software solution are:

- Portability of the Braille Device: Since no interface card has to be utilized, it is relatively easy to operate a Braille display with different computers.

- Possibility to use with a notebook PC

- Portability: Since it is still hardly possible to integrate an interface card into a portable PC, a software solution is required to employ a Braille display for such a computer. 


\subsection{The Hardware Solution}

In the PC, there is installed an interface card that is connected to the Braille display and that is furnished with a special firmware that monitors the Video Memory and sends its contents out to the Braille display. The chief advantages of the hardware solution are the following:

- Considerable Independence from the Underlying Operating System

- No Memory Occupied by the Driver Software

- No Conflicts With Other TSR's

\section{Braille Displays and Graphical User Interfaces}

It is completely obvious that a conventional one-line Braille display is not suited to furnish a tactile representation of a screen in graphics mode. However, in the last two years the development of software products was begun that make certain classes of graphically oriented environments accessible to the blind by means of synthetic speech. This is done by means of so-called "Off Screen Models", these are textual descriptions of elements on a graphic screen. With such off screen models it is indeed possible to describe standard controls such as pull-down menus, list boxes, check boxes, radio buttons, etc., as they are common within Microsoft Windows or $\mathrm{OS} / 2$. Icons can be made accessible at least inasmuch as it is possible to assign a verbal label to an icon that is heard when the icon is met by the mouse pointer. Of course, this method completely fails when it is attempted to make maps, technical drawings or photos readable by a blind user.

Although, as already mentioned, most of these new programs were originally developed with a speech synthesizer as an output medium in mind, presently a strong trend can be felt to adapt them for a Braille display afterwards.

In doing so, some difficulties have to be overcome, many of which arise from the significant differences between a speech and a Braille system: While in a speech system the various items of information are conveyed sequentially - one item after another -, the information presented by a Braille display is in a sense twodimensional: Although there is only one physical display line, the blind user sees the screen contents line by line, and, even more important, (s)he gets a complete tactile image of the geometrical arrangement of items within one line. In many cases, this image can be extended much beyond the current line.

Such geometrical views of the screen are nearly illusive to the user of a synthetic speech device: Although most speech-based screen review programs incorporate facilities to retrieve the screen coordinates of a character, these tools are much harder to use than the parallel ones on a Braille display.

This problem has, at least at present, a very negative impact on Braille software for GUI's: Since so little geometrical information is immediately encompassed within a speech-oriented GUI reviewer, the same is true for the Braille versions of these products. There have to be undertaken considerable efforts to make Braille-based GUI review software as "geometrically aware" as their textual counterparts are.

To work toward this aim, an extended, Braille-oriented concept of an Off Screen Model seems to be necessary. 